\title{
The ethical and moral responsibility of the surgeon, hospital and government for the hospitalization after adenotonsillectomy
}

\author{
Codrut Sarafoleanu ${ }^{1,2,3}$, Violeta Melinte ${ }^{1,2,3}$, Gabriela Musat ${ }^{1,3}$ \\ ${ }^{1}$ ENT\&HNS Department, "Sfanta Maria" Hospital, Bucharest, Romania \\ ${ }^{2}$ CESITO Center, "Sfanta Maria” Hospital, Bucharest, Romania \\ "Carol Davila" University of Medicine and Pharmacy, Bucharest, Romania
}

\begin{abstract}
Adenotonsillectomy is one of the most performed surgical procedures in otorhinolaryngology. When it comes to hospitalization duration, there are no general available truths. Every patient is unique and only the physician should decide for how long he/ she has to remain in the hospital after adenotonsillectomy, taking into consideration the used technique, the intraoperatory evolution and also the associated risk factors.

The authors present some general aspects of this theme and their own point of view in what adenotonsillectomy is concerned in terms of ethics and moral responsibility of the surgeon, hospital and government for the hospitalization after the surgical intervention.
\end{abstract}

KEYWORDS: ethics, moral responsibility, adenotonsillectomy, hospitalization.

\section{INTRODUCTION}

Regarding the interaction between the physician and the patient, the ethical and moral responsibility of the surgeon is one of the attributes that confer the patient a good and healthy experience. But, when the hospital management and the governmental laws come into discussion, things get complicated and it is not easy to make the best decisions.

Today, deontology is dictated by the law and led by the judges. The laws of the sanitary democracy represent the transition from the "idyllic" society to the developed society and establish the relationship authorities - physician - patient.
HOSPITALIZATION AFTER

\section{ADENOTONSILLECTOMY - ETHICAL AND MORAL RESPONSIBILITY}

Ethical and moral responsibility has an important role in each and every aspect of medical practice, but most of all when it comes to surgical indications. From our point of view, it is ethical and, last but not least, moral to establish a realistic surgical indication for every type of pathology and patient.

If we follow the guidelines and the studies found in the literature, in what adenotonsillectomy is concerned, there are absolute and relative indications, which can vary according to our patient's 
age $^{1}$. Absolute indications consist in: severe dysphagia, cor pulmonale, failure to thrive, adenotonsillar hyperplasia with obstructive sleep apnea or abnormal dentofacial growth, suspicion of malignant disease and (for tonsillectomy) hemorrhagic tonsillitis. Speech impairment, halitosis, adenotonsillar hyperplasia with upper airway obstruction are relative indications for adenotonsillectomy. Simple adenoidectomy is a relative indication for otitis media with effusion, recurrent or chronic rhinosinusitis or adenoiditis. When it comes to peritonsillar abscess, chronic or recurrent pharyngotonsillitis and streptococcal carriage, tonsillectomy alone might be performed ${ }^{2}$.

During the years, many discussions appeared regarding the hospitalization duration. While in the USA and in Western Europe it is thought that patients' quality of life is improved by a shorter stay in the hospital, in Romania the optimal medium hospitalization duration is considered to be 5 days.

In what adenotonsillectomy is concerned, hospitalization for more than 24 hours after the surgical intervention is still controversial.

In the USA, outpatient tonsillectomy and/or adenoidectomy is the procedure of choice, especially in patients with minimum complication risk.

In Europe, the transition between the traditional duration of hospital stay and same-day discharge slowly increases. A prospective 8-year study performed 18 years ago by Rivas Lacarte, in Spain, made the comparison between tonsillectomy in outpatients and inpatients. The study concluded that patients can be discharged 4 hours after the surgical intervention, because there is a slow rate of complications after tonsillectomy ${ }^{3}$. The authors also studied the influence of age (children under 4 years), sleep apnea syndrome and the complication risk and they did not find any relationship?.

In Romania, most hospitals' policy considers tonsillectomy alone or in combination with adenoidectomy to be an inpatient procedure, with a hospitalization duration from 24 to 48 hours. There are a few arguments that sustain this policy, such as immediate and short-term complications that can arise after surgery, like haemorrhage, apnea and infections, poor oral intake (with need for consecutive i.v. fluid supply), fever and protracted vomiting ${ }^{4}$. Patients that undergo surgery because of severe obstructive symptoms should be kept under observation by both the otorhinolaryngologist and anaesthesiologist because they have significantly more sequelae risk than those indicated for chronic or recurrent infections. Also, patients with underlying general diseases, postoperative bleeding and poor compliance should be maintained in hospital for at least 24 hours. Not least, there are patients or parents who request a prolonged admission.

Even though the complications after adenotonsillectomy are rare, bleeding is the most serious one that can appear and it can lead to a tragic end individually. The first spike in the bleeding event was found within the first 24 hours after surgery. A second spike seems to occur between the $5^{\text {th }}$ and the $7^{\text {th }}$ postoperative day and another after the $8^{\text {th }}$ and the $15^{\text {th }}$ postoperative day. According to the DRGs, a postoperative stay in the hospital for more than 6 days is difficult to be achieved ${ }^{5}$.

At the same time, there are arguments that sustain the outpatient procedure. Most trials that argue for a same-day discharge take the low percentage of postoperative haemorrhage into account. Age, general risk of postoperative haemorrhage and distance to the hospital are not enough criteria to indicate inpatient observation. Also, the costs for each patient rise directly proportionally to the staying duration; this is why each hospital has its own policy regarding the number of hospitalization days for adenotonsillectomy. We should not forget that the government policy also has an involvement in taking the decision about the hospitalization length.

\section{PATIENTS VERSUS HOSPITAL MANAGERS VERSUS PHYSICIANS POINTS OF VIEW}

Depending on who analyzes this problem, there seem to be different opinions about the subject and each category judgement is influenced by very different types of reasoning more or less moral or ethical.

In 2004, Wahlers ${ }^{6}$ was interested in patients' point of view regarding hospitalization after tonsillectomy, so he developed a questionnaire for them. The conclusion was that most of the patients $(79.6 \%)$ wanted to be kept under observation in hospital for about 4 days postoperatively, firstly for an optimal analgesic treatment and secondarily in order to diminish the postoperative bleeding risk. None of the patients who filled in the questionnaire checked the option of a 24hour or less hospitalization.

Another article based on patients' preferences regarding the length of hospital accommodation and published in Norway by Soreide and Olofsson emphasized that $92 \%$ of the interviewed participants considered tonsillectomy eligible for a day case procedure ${ }^{7}$.

After patients' point of view, we bring into discussion the hospital managers' idea concerning the hospitalization after adenotonsillectomy. Led by managing principles such as costs and different statistics regarding postoperative complications, a 
managing director of a hospital said: "There is no need for hospitalization and patients can return home a few hours after surgery; nine out of ten patients do well enough to go home".

Analyzing this reaction, the questions one should ask have to be the following:

Is this attitude compatible with moral and ethical responsibility?

Is it enough to experience a potential fatal complication once to change this opinion or the only leading principle is the cost?

Last but not least, the physicians' point of view is different depending on various factors. According to Wright et al. ${ }^{8}$, the indications for inpatient monitoring should include the following:

1. Age younger than 3 .

2. Obstructive sleep apnea or craniofacial syndromes involving the airway.

3. Systemic disorders which would put the patient at increased perioperative risk.

4. Poor socioeconomic status or other situation which would limit the patient's ability to return quickly to the hospital.

5 . When the procedure is done for a peritonsillar abscess.

6. Those who experience vomiting or haemorrhage.

Talking from the physician's point of view, tonsillectomy and adenoidectomy can be performed using various types of procedures and the hospitalization duration should be established also depending on the surgical procedure type used by the ENT doctor.

Traditional methods of tonsillectomy include the use of a scalpel and scissors, which can cause considerable bleeding during and after the surgical intervention.

Electrosurgery has provided improvements in surgical technique by decreasing operating time and improving haemostasis. Compared to cold dissection, there is no difference in postoperative haemorrhage rates, but electrosurgery increases pain ${ }^{9}$.

In case of coblation tonsillectomy, compared to the traditional procedures, patients report less pain, less necessity of narcotic pain medication and much quicker return to a regular diet and normal daily activities ${ }^{10}$.

Intracapsular power-assisted partial tonsillectomy produces less postoperative pain and fewer episodes of delayed haemorrhage and dehydration, but the effectiveness is the same as standard tonsillectomy in relieving obstructive sleep-disordered breathing ${ }^{11}$.

The harmonic scalpel uses ultrasonic technology to cut and coagulate tissues at lower temperatures. There is no evidence of important differences be- tween the use of the harmonic scalpel and other tonsillectomy techniques regarding postoperative haemorrhage ${ }^{12}$.

LASER-assisted tonsillectomy has shown less intraoperative bleeding and shorter operative time. According to Wan Ishlah, in the hospital where a laser machine and expertise are available, it is justifiable to use this technique as an effective method of performing tonsillectomy ${ }^{13}$.

From an otorhinolaryngologist's point of view, the determinant factors for establishing the duration of hospitalization should be represented by: the type of surgical procedure, local anatomic conditions, general associated diseases, the surgeon's option and experience, intraoperatory complications, patients' requirement. Most of all, adenotonsillectomy should be performed only when it is believed to provide enough benefit to justify doing the surgery.

In our opinion, ethics should break any administrative barrier and no restrictions should be related to the hospital or government policy if there are medical arguments for a longer hospitalization. Different opinions can be found worldwide regarding hospitalization after adenotonsillectomy and medico-legal aspects should be taken into account when deciding patient discharge.

\section{CONCLUSIONS}

Adenotonsillectomy with hospitalization remains one of the most performed procedures in otorhinolaryngology, but its frequency trends to decrease due to the new available surgical techniques with less intra- and post-operative complications.

The duration of hospitalization is still debatable and different worldwide, but the physician should be the one who decides for how long the patients remain under observation, taking into considerations all the risk factors, because he is the first one who has ethical and moral responsibility not only in front of the patient, but also in front of the law.

Various factors influence the duration of hospitalization and the most important seems to be the type of procedure, special conditions related to the case and costs. Also, the medical insurance of the patient plays an important role.

Unfortunately, both ethical and moral aspects are sometimes neglected or underestimated, the doctor - patient relationship being seriously affected.

Surgeons should be sufficiently cautious to avoid fatal events and complications, self-related ethical and moral problems, medico-legal issues, independent of hospital and government policy. Patients' life is above all administrative policies. 
Conflict of interest: The authors have no conflict of interest.

Contribution of authors: All authors have equally contributed to this work.

\section{REFERENCES}

1. Darrow DH, Siemens C. Indications for tonsillectomy and adenoidectomy. Laryngoscope. 2002;112(8 Pt 2 Suppl 100):6-10. DOI: 10.1002/lary.5541121404.

2. Hoddeson EK, Gourin CG. Adult tonsillectomy: current indications and outcomes. Otolaryngol Head Neck Surg. 2009;140(1):19-22. DOI: 10.1016/j.otohns.2008.09.023.

3. Rivas LM. Tonsillectomy as a major outpatient procedure. Prospective 8-year study: indications and complications. Comparison with inpatients. Acta Otorrinolaringol Esp. 2000;51(3):221-7.

4. Randall DA, Hoffer ME. Complications of tonsillectomy and adenoidectomy. Otolaryngol Head Neck Surg. 1998;118(1):61-8.

5. Negm H, Atef A, Lasheen H, Kamel AA, Azooz K, Elhoussainy O. Factors affecting secondary post-tonsillectomy hemorrhage: a casecontrol study. Egypt J Otolaryngol. 2017;33(1):50-5.

6. Wahlers A. Inpatient treatment of tonsillectomy from the patient's point of view. HNO. 2004;52(2):153-5.

7. Søreide AK, Olofsson J. Tonsillectomy--day surgery or hospitalization? Tidsskr Nor Laegeforen. 1999;119(10):1423-7.

8. Wright S, Deskin R. Tonsillitis, Tonsillectomy, and Adenoidectomy. In: Quinn FB Jr., Ryan MW. Grand Rounds Presentation, UTMB, Dept. of Otolaryngology, University of Texas Medical Branch; 2003. Available from: http://www.utmb.edu/otoref/grnds/Tons-20031105/Tons-2003-1105.htm.

9. Mowatt G, Cook JA, Fraser C, McKerrow WS, Burr JM. Systematic review of the safety of electrosurgery for tonsillectomy. Clinical Otolaryngology. 2006;31 (2):95-102. DOI: 10.1111/j.1749-4486.2006.01162.x.

10. Omrani M, Barati B, Omidifar N, Okhovvat AR, Hashemi SA. Coblation versus traditional tonsillectomy: A double blind randomized controlled trial. J Res Med Sci. 2012;17(1):45-50.

11. Koltai PJ, Solares CA, Mascha EJ, Xu M. Intracapsular partial tonsillectomy for tonsillar hypertrophy in children. Laryngoscope. 2002;112(8 Pt 2 Suppl 100):17-9. DOI: 10.1002/lary.5541121407.

12. Neumann C, Street I, Lowe D, Sudhoff H. Harmonic scalpel tonsillectomy: a systematic review of evidence for postoperative hemorrhage. Otolaryngol Head Neck Surg. 2007;137(3):378-84. DOI: 10.1016/j.otohns.2007.05.003.

13. Ishlah LW, Fahmi AM, Srinovianti N. Laser versus dissection technique of tonsillectomy. Med J Malaysia. 2005;60(1):76-80. 